\title{
Some Ground-State Properties of a Binary Boson Mixture
}

\author{
Tapash Chakraborty* \\ Institut für Theoretische Physik der Universität zu Köln, Köln, West Germany
}

(Received September 18, 1981; revised January 25, 1982)

The optimal Jastrow wave function in the hypernetted-chain scheme is shown to generate numerically suitable expressions for the pressure and the compressibility of a binary boson mixture. In the single-component case, spin-polarized tritium is shown to be unstable for densities below $0.0044 \AA^{-3}$.

\section{INTRODUCTION}

The microscopic theory for single-component Bose fluids (e.g., ${ }^{4} \mathrm{He}, \mathrm{H} \uparrow$ ) has avhieved great success in recent years. Various optimization procedures $^{1-4}$ have been devised to obtain the correct long-wavelength behavior of the structure functions which are in close agreement with recent experimental results. ${ }^{5}$ Generalization of these techniques for multicomponent systems has been achieved recently. ${ }^{6,7}$ The formalism has also been applied to the problem of two ${ }^{3} \mathrm{He}$ atoms in ${ }^{4} \mathrm{He}$ by Owen. ${ }^{8}$ In this paper, we will show that the optimization procedure developed in Ref. 7 generates numerically suitable expressions for the pressure and the compressibility of binary boson mixtures.

In Section 2, we derive the pressure and the compressibility equations, which are then applied to the isotopic mixture of spin-polarized tritium (T $\uparrow)$ and hydrogen $(\mathrm{H} \uparrow)$. The ground state properties of pure $\mathrm{T} \uparrow$ and $\mathrm{H} \uparrow$ are also given. For pure $T \uparrow$, a particular density is obtained below which the uniform system is found to be unstable.

\footnotetext{
*Present address: Department of Theoretical Physics, University of Oulu, Linnanmaa, Oulu, Finland.
} 


\section{THEORY}

Let us begin with the Jastrow-type wave function for the ground state

$$
\begin{aligned}
& \Psi\left(\mathbf{r}_{1,1}, \ldots, \mathbf{r}_{1, N_{1}} ; \mathbf{r}_{2,1}, \ldots, \mathbf{r}_{2, N_{2}}\right) \\
& =\exp \frac{1}{2}\left[\sum_{i<j}^{N_{1}} u_{11}\left(\left|\mathbf{r}_{1, i}-\mathbf{r}_{1, j}\right|\right)+\sum_{i<j}^{N_{2}} u_{22}\left(\left|\mathbf{r}_{2, i}-\mathbf{r}_{2, j}\right|\right)\right. \\
& \left.\quad+\sum_{\substack{i=1, N_{1} \\
j=1, N_{2}}} u_{12}\left(\left|\mathbf{r}_{1, i}-\mathbf{r}_{2, j}\right|\right)\right]
\end{aligned}
$$

where $u_{\alpha \beta}(r)$ are the correlation functions for the particle pairs (11), (22), and (12), and $N_{1}$ and $N_{2}$ are the numbers of the two species in the uniform system. Defining the radical distribution functions

$$
\rho_{\alpha} \rho_{\beta} g_{\alpha \beta}\left(r_{i_{\alpha}, i_{\beta}}\right)=N_{\alpha}\left(N_{\beta}-\delta_{\alpha \beta}\right) \int|\Psi|^{2} d \mathbf{r}_{\left(i_{\alpha}, i_{\beta}\right)}
$$

where $\int|\Psi|^{2} d \mathbf{r}_{12 \cdots N}=1$, and $d \mathbf{r}_{\left(i_{\alpha}, i_{\beta}\right)}$ denotes $d \mathbf{r}_{12 \cdots N}$ with $i_{\alpha}$ and $i_{\beta}$ omitted, and employing the $\mathrm{HNC}$ formalism, ${ }^{2}$ we obtain the ground state energy for the binary mixture

$$
E=x_{1}^{2} E_{11}+x_{2}^{2} E_{22}+2 x_{1} x_{2} E_{12}
$$

with

$$
\begin{aligned}
E_{\alpha \beta}= & \frac{\hbar^{2} \rho}{8 m_{\alpha \beta}} \int g_{\alpha \beta}^{-1}\left(\nabla g_{\alpha \beta}\right)^{2}+\frac{1}{2} \rho \int g_{\alpha \beta}(r) v_{\alpha \beta}(r) d \mathbf{r} \\
& -\frac{\hbar^{2} \rho}{8 m_{\alpha \beta}} \frac{\delta_{\alpha \beta}}{(2 \pi)^{3} \rho_{\alpha} \rho_{\beta}} \int\left[S_{\alpha \beta}\left(S_{\alpha \beta}-3\right)-\frac{S_{\gamma \gamma}}{D}+S_{\alpha \gamma}^{2}+3\right] k^{2} d \mathbf{k} \\
m_{\alpha \beta}^{-1}= & 0.5\left(m_{\alpha}^{-1}+m_{\beta}^{-1}\right), \quad \alpha \neq \beta, \quad D(k)=S_{11}(k) S_{22}(k)-S_{12}^{2}(k)
\end{aligned}
$$

Here $x_{\alpha}=\rho_{\alpha} / \rho$ is the concentration of the species $\alpha$, while $S_{\alpha \beta}(k)$ are the structure functions,

$$
S_{\alpha \beta}(k)-\delta_{\alpha \beta}=\left(\rho_{\alpha} \rho_{\beta}\right)^{1 / 2} \int\left[g_{\alpha \beta}(r)-1\right] \exp (i \mathbf{k} \cdot \mathbf{r}) d \mathbf{r}
$$

Minimization of the energy with respect to the radical distribution functions results in the following Euler-Lagrange equations ${ }^{7}$ :

$$
\left[-\left(\hbar^{2} / m_{\alpha \beta}\right) \nabla^{2}+v_{\alpha \beta}(r)+W_{\alpha \beta}(r)\right] g_{\alpha \beta}^{1 / 2}(r)=0
$$

Here $v_{\alpha \beta}(r)$ are the interaction potentials, and the induced potentials $W_{\alpha \beta}(r)$ 
are given in the Fourier space as

$$
\begin{aligned}
& W_{\alpha \alpha}(k)=-\frac{\hbar^{2} k^{2}}{4 \rho_{\alpha}}\left[m_{\alpha}^{-1}\left(2 S_{\alpha \alpha}-3+\frac{S_{\beta \beta}^{2}}{D^{2}}\right)+m_{\beta}^{-1} \frac{S_{\alpha \beta}^{2}}{D^{2}}\right] \\
& W_{\alpha \beta}(k)=-\frac{\hbar^{2} k^{2}}{4\left(\rho_{\alpha} \rho_{\beta}\right)^{1 / 2}}\left[2 m_{\alpha \beta}^{-1} S_{\alpha \beta}-\frac{S_{\alpha \beta}\left(m_{\alpha}^{-1} S_{\beta \beta}+m_{\beta}^{-1} S_{\alpha \alpha}\right)}{D^{2}}\right]
\end{aligned}
$$

Once the optimum distribution functions $g_{\alpha \beta}(r)$ are obtained, the density derivative of $g_{\alpha \beta}(r)$ can be neglected when we differentiate (3) with respect to $\rho$. The pressure equation is then given by

$$
\begin{aligned}
\frac{P}{\rho} & =\rho\left(\frac{\partial E}{\partial \rho}\right)_{x_{1}} \\
& =E-\int \frac{d \mathbf{k}}{(2 \pi)^{3}} \sum_{\alpha \neq \beta} \frac{\hbar^{2} k^{2}}{8 m_{\alpha} \rho}\left[S_{\alpha \alpha}+\frac{1+3 S_{\beta \beta}}{D}-\frac{S_{\beta \beta}\left(S_{\alpha \alpha}+S_{\beta \beta}\right)}{D^{2}}-3\right]
\end{aligned}
$$

The integrand in (7) depends only on the structure functions, which can be calculated very accurately from (5) and (6). The "thermodynamic" pressure thus obtained is formally equivalent to the pressure from the "virial theorem.",

From (5), the calculation of compressibility is straightforward. This is given by

$$
\kappa^{-1} / \rho=C=d P / d \rho
$$

with

$$
\begin{aligned}
C= & E+\rho \frac{\partial E}{\partial \rho} \\
& -\sum_{\alpha \neq \beta} \int \frac{d \mathbf{k}}{(2 \pi)^{3}} \frac{\hbar^{2} k^{2}}{8 m_{\alpha}}\left[\left(1-\frac{3 S_{\beta \beta}^{2}}{D^{2}}+\frac{2 S_{\beta \beta} S_{\alpha \beta}^{2}}{D^{3}}+\frac{2 S_{\beta \beta}^{3}}{D^{3}}\right) \frac{d S_{\alpha \alpha}}{d \rho}\right. \\
& +\left(\frac{2 S_{\alpha \beta}^{2}\left(S_{\alpha \alpha}+S_{\beta \beta}\right)}{D^{3}}-\frac{3 S_{\alpha \beta}^{2}}{D^{2}}\right) \frac{d S_{\beta \beta}}{d \rho} \\
& \left.+\left(\frac{2 S_{\beta \beta} S_{\alpha \beta}\left(3-2 S_{\beta \beta} / D\right)}{D^{2}}-\frac{2 S_{\alpha \beta}\left(S_{\alpha \alpha} S_{\beta \beta}+S_{\alpha \beta}^{2}\right)}{D^{3}}\right) \frac{d S_{\alpha \beta}}{d \rho}\right]
\end{aligned}
$$

To evaluate the integrals in (8) one needs to know $d S_{\alpha \beta} / d \rho$. This can be 
obtained by differentiating (5), which results in

$$
\begin{aligned}
& \left(\frac{\hbar^{2}}{m_{\alpha \beta}} \nabla^{2}-v_{\alpha \beta}-W_{\alpha \beta}\right) y_{\alpha \beta} \\
& \quad+g_{\alpha \beta}^{1 / 2} \int \sum_{i j} V_{i j}^{\alpha \beta}\left(\mathbf{r}-\mathbf{r}^{\prime}\right) g_{i j}^{1 / 2}\left(r_{i j}^{\prime}\right) y_{i j}\left(r_{i j}^{\prime}\right) d \mathbf{r}^{\prime} \\
& =-g_{\alpha \beta}^{1 / 2}(r) \mathscr{F}\left[W_{\alpha \beta}^{\prime}\right] \\
& V_{i j}^{\alpha \beta}(k)=\partial W_{\alpha \beta}(k) / \partial S_{i j}(k), \quad \alpha, \beta=1,2
\end{aligned}
$$

where $\mathscr{F}[f]$ denotes the Fourier transform of $f, y_{\alpha \beta} \equiv \partial g_{\alpha \beta}^{1 / 2} / \partial \rho$, and

$$
\begin{aligned}
\rho^{2} x_{\alpha} W_{\alpha \alpha}^{\prime}(k) & \\
= & {\left[\frac{\hbar^{2} k^{2}}{4 m_{\alpha}}\left(1-\frac{3 S_{\beta \beta}^{2}}{D^{2}}+\frac{2 S_{\beta \beta} S_{\alpha \beta}^{2}}{D^{3}}+\frac{2 S_{\beta \beta}^{3}}{D^{3}}\right)\right.} \\
& \left.+\frac{\hbar^{2} k^{2}}{4 m_{\beta}}\left(\frac{2 S_{\alpha \beta}^{2}\left(S_{\alpha \alpha}+S_{\beta \beta}\right)}{D^{3}}-\frac{3 S_{\alpha \beta}^{2}}{D^{2}}\right)\right] \\
\rho^{2}\left(x_{\alpha} x_{\beta}\right)^{1 / 2} W_{\alpha \beta}^{\prime}(k) & \\
= & {\left[\frac{\hbar^{2} k^{2}}{4 m_{\alpha}}\left(\frac{S_{\beta \beta} S_{\alpha \beta}\left(3-2 S_{\beta \beta} / D\right)}{D^{2}}-\frac{S_{\alpha \beta}\left(S_{\alpha \alpha} S_{\beta \beta}+S_{\alpha \beta}^{2}\right)}{D^{3}}\right)\right.} \\
& \left.+\frac{\hbar^{2} k^{2}}{4 m_{\beta}}\left(\frac{S_{\alpha \alpha} S_{\alpha \beta}\left(3-2 S_{\alpha \alpha} / D\right)}{D^{2}}-\frac{S_{\alpha \beta}\left(S_{\alpha \alpha} S_{\beta \beta}+S_{\alpha \beta}^{2}\right)}{D^{3}}\right)\right]
\end{aligned}
$$

The pressure and compressibility equations given above are suitable for an accurate numerical evaluation of those quantities. They are also useful for analysis of the structure of the solutions of the Euler-Lagrange equations. Performing the second variation of the energy with respect to $\delta g_{\alpha \beta}^{1 / 2}$ and relating the condition that minimizes the energy to the compressibility, one obtains the stability condition ${ }^{9}$ for $g_{\alpha \beta}^{1 / 2}$. An analysis of this type has been completed for binary boson mixtures and will be published elsewhere. ${ }^{10}$

\section{RESULTS}

We have solved the above equations for the isotopic mixture of $\mathrm{T} \uparrow$ and $\mathrm{H} \uparrow$. These new quantum systems are very much of current interest. ${ }^{11,12}$ It has been predicted ${ }^{12}$ that, because of the small mass and weak interaction, the $\mathrm{H} \uparrow$ system will be a Bose gas down to ansolute zero, while $\mathrm{T} \uparrow$ will be a self-bound liquid very much like ${ }^{4} \mathrm{He}$. [In fact, the values of the "quantum 


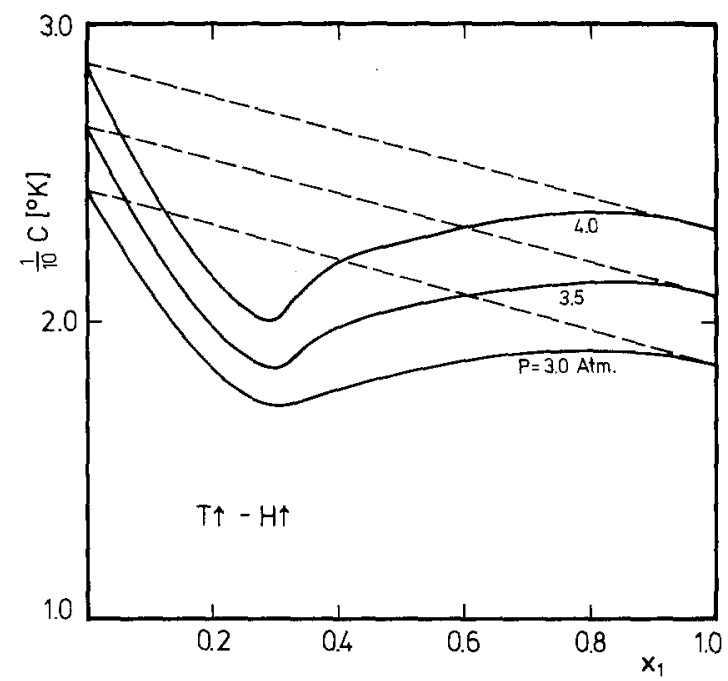

Fig. 1. Compressibility as a function of $\mathrm{H} \uparrow$ concentration for the isotopic mixture $\mathrm{T} \uparrow-\mathrm{H} \uparrow$.

parameter" ${ }^{, 12} \eta$ for $\mathrm{T} \uparrow(0.183)$ and ${ }^{4} \mathrm{He}(0.1815)$ are very close.] The interaction potentials for these systems are very accurately known. ${ }^{13}$ The $\mathrm{T} \uparrow-\mathrm{H} \uparrow$ mixture is, however, known to phase-separate completely at all pressures and concentrations. ${ }^{7}$ In Fig. 1 , we plot $C$ for the mixture as a function of $\mathrm{H} \uparrow$ concentration for $P=3.0,3.5$, and $4.0 \mathrm{~atm}$. The dashed lines represent the ideal solution $C=x_{1} C_{1}+x_{2} C_{2}$, with $C_{1}$ and $C_{2}$ the compressibilities of the pure components $\mathrm{T} \uparrow$ and $\mathrm{H} \uparrow$. In the singlecomponent case, (5)-(8) reduce to the equations derived earlier. ${ }^{3,4}$ These equations have been used to study the ground-state properties ${ }^{11}$ of $\mathrm{H} \uparrow$. The results are given in Fig. 2 for the sake of completeness. As expected, the energy is always positive and the pressure and compressibility go to zero at zero density. In Fig. 2, we also present the results in this case is that at the density $\rho_{c}=0.0044 \AA^{-3}$, the compressibility vanishes, rendering the uniform system unstable with respect to density fluctuations. It is worth noting that a similar phenomenon was observed ${ }^{4}$ in ${ }^{4} \mathrm{He}$, and subsequent formal analysis ${ }^{9}$ has provided evidence that this is a consequence of the long-range behavior known to be present in these systems with short-range forces. Due to the low-density regime of $\mathrm{T} \uparrow$ as compared to ${ }^{4} \mathrm{He}$, we expect that the present calculation quite accurately pinpoints $\rho_{c}$. At the density $\rho_{c}$, the phonon screening in the medium disappears $\left(C=m s^{2}\right.$, where $s$ is the speed of sound), and

$$
S^{\prime}(0)=-\rho \pi^{2} \lim _{r \rightarrow \infty}\left\{r^{4}[g(r)-1]\right\}
$$




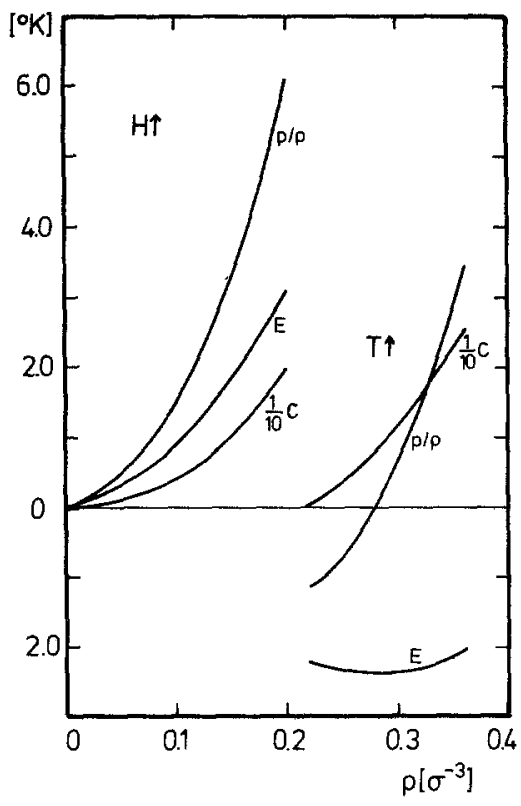

Fig. 2. Energy, pressure, and compressibility of $\mathrm{H} \uparrow$ and $\mathrm{T} \uparrow$. Here $\sigma=3.69 \AA^{-3}$.

becomes infinite. Here $g(r)$ and $S(k)$ are the single-component functions. The energy vs. density curve is very flat around the equilibrium density $\left(\rho_{0}=0.0056 \AA^{-3}\right)$, where the pressure is zero.

In conclusion, the optimization procedure ${ }^{7}$ for binary boson mixtures is shown to generate expressions for the pressure and compressibility which are suitable for numerical work. The correct long-range behavior of systems with short-range forces can be obtained through optimization techniques. Similar study of the long-range behavior in the mixture is in progress. The mixture formalism has been applied to a mixture of charged particles, ${ }^{10}$ where the long-range Coulomb forces play the dominant role. ${ }^{14,15}$ The results for these systems are reserved for separate publication.

\section{ACKNOWLEDGMENTS}

I wish to thank Prof. M. L. Ristig for his kind help and interest, and Dr. Stefano Fantoni for many helpful discussions.

\section{REFERENCES}

1. C. E. Campbell, in Progress in Liquid Physics, C. A. Croxton. ed. (Wiley, New York, 1978), Chapter 6.

2. K. Hiroike, Prog. Theor. Phys. 24, 317 (1960). 
3. L. J. Lantto, A. D. Jackson, and P. J. Siemens, Phys. Lett. 68B, 311 (1977).

4. A. D. Jackson, A. Lande, and L. J. Lantto, Nucl. Phys. A 317, 70 (1979).

5. R. B. Hallcock, Phys. Rev. A 5, 320 (1972).

6. C. E. Campbell, Ann. Phys. 73, 43 (1972).

7. T. Chakraborty, Phys. Rev. B, in press.

8. J. C. Owen, Phys. Rev. Lett. 47, 586 (1981).

9. L. Castillejo, A. D. Jackson, B. K. Jennings, and R. A. Smith, Phys. Rev. B 20, 3631 (1979).

10. T. Chakraborty, Phys. Rev. B, submitted.

11. L. J. Lantto and R. M. Nieminen, J. Low Temp. Phys. 37, 1 (1979).

12. L. H. Nosanow, J. Phys. (Paris) 41 C7 (1980).

13. W. Kolos and L. Wolniewicz, J. Chem. Phys. 43, 2429 (1965).

14. W. H. Bassichis, Phys. Rev. 134, A543 (1964).

15. J. P. Hansen, R. Mazighi, and P. Vieillefosse, Phys. Lett. 81A, 215 (1981). 